Research Paper:

\title{
Predictors of Health-Promoting Behaviors Among Kurdish Healthcare Providers in Iraqi Kurdistan
}

\author{
Aram Salih Mohammed Amin Kamali ${ }^{1}$, Roya Sadeghi²*, Azar Tol${ }^{2}$, Mahdi Yaseri ${ }^{3}$ \\ 1. Department of Health Promotion and Education, School of Public Health, International Campus, Tehran University of Medical Sciences, Tehran, Iran. \\ 2. Department of Health Promotion and Education, School of Public Health, Tehran University of Medical Sciences, Tehran, Iran. \\ 3. Department of Epidemiology and Biostatistics, School of Public Health, Tehran University of Medical Sciences, Tehran, Iran.
}

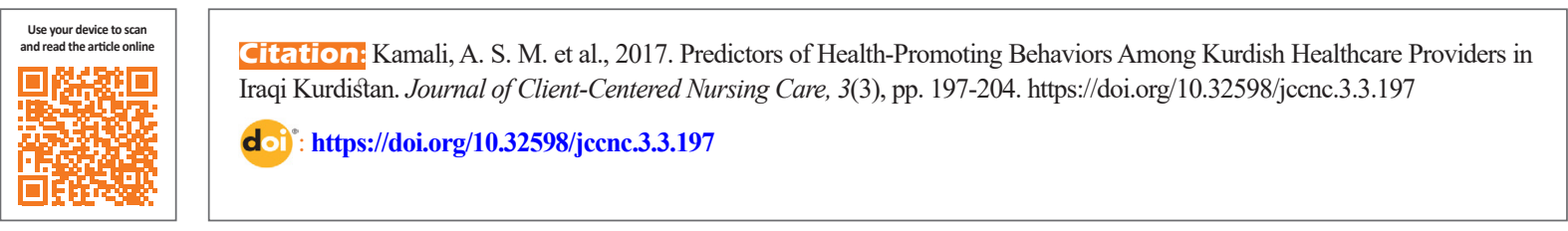

Article info:

Received: 06 Jan. 2017

Accepted: 19 May 2017

Keywords:

Health-promoting lifestyle, Healthcare providers, Noncommunicable diseases

\begin{abstract}
A B S T RA C T
Background: Health-promotion is universally accepted as the effective method for modifying the behavior of individuals towards ideal health. Thus, the present cross-sectional predictive study determined the predictors of health-promoting behaviors among Kurdish Healthcare Providers in Iraqi Kurdistan.

Methods: Demographic data were collected from a sample of 460 healthcare providers instead of the standard questionnaire of health-promoting lifestyle profile-II. These data were analyzed using descriptive statistics (mean and frequency) as well as inferential statistics including KST, t-test, Mann-Whiney test, ANOVA, and Wilcoxon sign-rank test using SPSS version 23.

Results: Approximately $55 \%$ of the participants were male, and $67.4 \%$ were married. In addition, $>72.5 \%$ of the participants had a family comprising of at least four members. Although $42 \%$ of the participants had $<5$ years of work experience, an $82.1 \%$ of the current study population held a Diploma. Moreover, $45.4 \%$ of the healthcare providers exhibited satisfaction with their income. The current study revealed that healthcare providers perceived that the health status was significantly predictive of the nutritional behavior and physical activity to gender $(\mathrm{P}=0.002, \mathrm{P}=$ 0.003 , respectively), spiritual growth to education level (0.014) and income satisfaction (0.02), as well as, interpersonal relation to expertise, (0.046), and work experience (0.036). Among all the samples scored, physical activities were found to be the lowest, while the spiritual growth was the highest.

Conclusion: The results illustrated that the participants do not undergo the complete healthpromoting behaviors, especially, physical activity. However, these behaviors play a critical role in health maintenance as well as improving the quality of life. Hence, the provision of strategies, including those in accordance with predictors of health-promoting behaviors, is vital for improving the health status of the healthcare providers.
\end{abstract}




\section{Background}

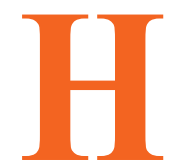

ealth-promoting activities are ordinarily accepted as encouraging lifestyle practices within society (Wallace 2000). The health improvement concept includes healthy lifestyle behavior defined by different methods. According to Tapp and Goldenthal, a healthy lifestyle should include both health promotion and disease prevention (Lee \& Wang, 2005). Walker defined the Health-Promoting Lifestyle (HPL) by a multidimensional pattern of self-initiated actions and perceptions that seek superior reserve or improve the level of wellness, self-actualization, and fulfillment in an individual (Sousa et al. 2015). This phenomenon is classified into six dimensions: Nutrition Behavior (NB: focuses on meal arrangements to produce healthy and balanced diet), Physical Activity (PA: measures regular exercise patterns and recreational activities), Stress Management (SM: quantifies the ability to cope with stress and focuses on relaxation techniques to control stress), Health Responsibility (HR: related to the individual's general health and well-being), Spiritual Growth (SG: broadly discusses an individual's attitude regarding life in general and the ability to believe in a creation greater then oneself), and Interpersonal Relationships (IR: related to a sense of intimacy and dealing with meaningful relationships) (Pender, Murdaugh \& Parsons 2002).

Since the healthcare providers play a positive role, it is crucial for the society to yield responsibility for their health. The educational and community-based programs focus on the healthy individuals (ECBP-HP, 2010) and emphasize the importance of preventing disease and improving the quality of life (Peker \& Bermek 2011). Although several worksites do not yet offer comprehensive health-promoting agendas, investigations have archived the ability of workplace programs for superior health outcomes. Funding, staff resources, inconvenient locations, limitation of times, and insufficient employee interest are the barriers to widespread implementation of health-promoting programs, especially, among high-risk employees (Schopp et al. 2015).

Our health is affected by the lifestyle, and unhealthy lifestyle and behavior play a specific role in the progression of chronic diseases (Scott \& Happell 2011). Non-Communicable Diseases (NCD) pose a significant burden considering its contributions toward mortality, morbidity, and quality of life; about 38 million people are deceased every year. Therefore, all age groups are being affected with NCDs (World Heaalth Organization 2015a), which is primarily linked to unhealthy lifestyle behaviors (Steyn \& Damasceno 2006).

The survey of NCD risk factors was conducted in Iraq (2006), which revealed that approximately $70 \%$ of the adult population was overweight and one-third was suffering from obesity; the obesity rate was found to be higher among females than males (World Heaalth Organization 2015b). Additionally, around $90 \%$ had low vegetable and fruit intake, while $57 \%$ performed less daily exercise (World Heaalth Organization 2015b). However, in comparison with the mortality and morbidity data, we found no information regarding health risk factors from Iraqi Kurdistan. Based on the experiences of demographically similar countries, the Kurdistan region could expect increased levels of chronic diseases as the population ages, urbanizes, and gains wealth.

Health promotion is universally accepted as the most effective method that assists the individuals in changing their behavior and shifting towards ideal health. Health-promoting activities can enhance and preserve the health of individuals. Therefore, to reach the goal of a healthy community requires health promotion as well as disease prevention (World Heaalth Organization 2005). The present study also aimed to explain the predictors of HPL among Kurdish Healthcare Providers (K.HcPrs) considering NB, SM, PA, HR, SG, and IR in order to compare their sociodemographic profile to those published from other studies using HealthPromoting Lifestyle Profile II (HPLP-II). Thus, we hypothesized that the demographic variable could predict each the HPLP-II constructs.

\section{Study design and setting}

This cross-sectional predictive study assessed HPLPII among K.HcPrs. It was conducted at the Directorate of Health, Garman administrative "Garman is located in the north of Iraq, Bordering with Iran in the west." Thus, the letter of permission was sent from the School of Public Health/Tehran University of Medical Sciences to Garman General Directorate of Health (GGDH), and from there to all hospitals and healthcare centers before conducting the study.

\section{Materials and Methods}

\section{Instruments}

We developed a demographic form instead of a questionnaire for obtaining the Socio-Economic Status (SES) and used the Kurdish version of HPLP-II (Kamali 
2015). This instrument consisted of 52 items encompassing the six aspects of health-promoting behaviors, as described above. It measured the respondent's selfreported health-promoting behaviors with high scores that indicated the frequent performance of the healthpromoting behaviors or habits of well-being on a fourpoint Likert scale $(1=$ Never, $2=$ Sometimes, $3=$ Often, $4=$ Routinely). The minimum and maximum HPLP-II scores are 52 and 208, respectively; the high score represents a maximal level of health with respect to lifestyle. The health-promoting lifestyle score is divided into four grades: poor (52-90), general (91-129), good (130-168), and excellent (169-208). The scores for each item were added for each subgroup and divided by the number of items in the subgroup to obtain the final subgroup scores. The total HPLP-II score was obtained by adding the scores for all the items and dividing by the total number of items. These scores are used as an index of the healthpromoting lifestyle: high score indicated a high level.

\section{Data collection and analysis}

The Kurdish version of HPLP-II was used to collect data from the K.HcPrs. We randomly distributed the selfadministered questionnaires to $500 \mathrm{HcPrs}$ in hospitals and healthcare centers during January 2016, of which, only $460 \mathrm{HcPrs}$ completed the study, thereby providing a $92 \%$ response rate.

For data analysis, the mean, standard deviation, and interquartile range were calculated. To the normal distribution of data was assessed using Kolmogorov-Smirnov test and Q-Q plot with an estimated $95 \%$ confidence interval. To compare the subgroups, t-test, Mann-Whiney test, ANOVA, and Kruskal-Wallis test were employed based on the normal distribution of data. The Wilcoxon sign-rank test was used for the analysis. The questionnaire was labeled and coded by the researchers and data reviewed for accuracy and completeness and analyzed using SPSS (IBM Co., SPSS Statistics for Windows, version 23.0. Armonk, NY, USA).

\section{Results}

The SES of the sample was described by the results obtained from the research questions. The participants included K.HcPrs in DoH/Garman Administrative, and approximately $55 \%$ were males. Furthermore, almost $49.3 \%$ of the participants were $<30$-year-old, while about $67.4 \%$ of the participants were married. In addition, $>72.5 \%$ of the participants had a family consisting of at least four members. Approximately, $39.4 \%$ participants were nurses. $42 \%$ of the participants had $<$
5 years' work experience. Also, a majority of our study population $(82.1 \%$ ) held a Diploma. $45.4 \%$ of HcPrs stated that they were pleased with their income (Table 1).

\section{Descriptive statistics for HPLP-II}

The mean (SD) of the overall HPLP-II questionnaire was 126.45 (16.91). The lowest mean (SD) in the subscales was 15.27 (4.79) for PA, while the highest was 24.47 (4.45) for SG (Table 2).

\section{SES related to HPLP-II}

The individual characteristics associated with HPL included the date of birth, gender, family size, marital status, expertise, job experience, education level, and income satisfaction.

The current study illustrated that NB was significantly correlated to gender $(\mathrm{P}=0.002)$, slightly significant with respect to age ( $\mathrm{P}=0.014)$, and just statistically significant to family size $(\mathrm{P}=0.054)$. On the other hand, $\mathrm{PA}$ was significantly associated with gender $(\mathrm{P}=0.003)$, and thus, significantly correlated with expertise $(\mathrm{P}=0.07)$. However, SG showed a statistically significant correlation between the education level $(\mathrm{P}=0.014)$ and income satisfaction $(\mathrm{P}=0.042)$. Conversely, $\mathrm{SG}$ illustrated demonstrated a slight significant correlation with marital status $(\mathrm{P}=0.056)$ and expertise $(\mathrm{P}=0.058)$. Moreover, IR was significantly correlated with expertise $(\mathrm{P}=0.046)$ and job experience $(\mathrm{P}=0.036)$, and it exhibited a slightly significant trend towards the level education $(\mathrm{P}=0.098)$. The mean scores of domains for each of the sub-criteria were shown in Table 3.

\section{Discussion}

Health-promoting behaviors have been investigated among the groups of various statuses. The socio-demographic variables such as university students (Ginsborg et al. 2009), adolescents (Chen, James \& Wang 2007), elderly (Kim 2009), women (Mirghafourvand et al. 2014), workers (Beser, Bahar \& Buyakkaya 2007), and academic staff (Pirincci et al. 2008) affect the HPL.

The mean value of K.HcPrs was recorded for health with respect to lifestyle based on the current method. In line with this study, Nacar et al. (2014) found general health situation among Turkish medical students.

Nevertheless, the involvement of healthcare providers was low in PA, moderate in the responsibilities towards their health and NB, and improved in SG, IR, and SM. 
Table 1. Description of socio-demographic variables

\begin{tabular}{|c|c|c|c|}
\hline Parameter & & $\mathbf{N}$ & $\%$ \\
\hline \multirow{3}{*}{ Age (Yeas) } & $\leq 30$ & 222 & 49.3 \\
\hline & $31-40$ & 144 & 31.9 \\
\hline & $41<$ & 85 & 18.8 \\
\hline \multirow{2}{*}{ Gender } & Male & 251 & 55.0 \\
\hline & Female & 205 & 45.0 \\
\hline \multirow{3}{*}{ Family size } & $\leq 3$ & 120 & 27.5 \\
\hline & 4 to 5 & 174 & 39.8 \\
\hline & $6<$ & 143 & 32.7 \\
\hline \multirow{3}{*}{ Marital status } & Single & 138 & 30.2 \\
\hline & Married & 308 & 67.4 \\
\hline & Divorce + Widow & 11 & 2.4 \\
\hline \multirow{4}{*}{ Expertise } & Doctor & 38 & 8.6 \\
\hline & Nurse & 175 & 39.2 \\
\hline & Laboratory specialist & 54 & 12.1 \\
\hline & Other specialist & 179 & 40.1 \\
\hline \multirow{3}{*}{ Job experience (years) } & $\leq 5$ & 167 & 42.0 \\
\hline & 6 to 10 & 82 & 20.6 \\
\hline & $11<$ & 149 & 37.4 \\
\hline \multirow{3}{*}{ Education level } & Diploma & 367 & 82.1 \\
\hline & Bachelor & 73 & 16.3 \\
\hline & MSc. + PhD & 7 & 1.6 \\
\hline \multirow{3}{*}{ Income satisfaction } & Satisfied & 155 & 35.0 \\
\hline & Somehow satisfied & 201 & 45.4 \\
\hline & Not satisfied & 87 & 19.6 \\
\hline
\end{tabular}

The measures of PA were the lowest and that of SG at the highest level. Thus, healthy behaviors could be considered at an average level, thereby demonstrating that PA exhibited the lowest score among all the dimensions of HPLP-II, while the SG dimension obtained the highest score according to some studies (Fahimen et al. 2007;

Table 2. Description of HPL profile -II's dimensions

\begin{tabular}{ccc} 
Dimensions & N. Questions & Mean \pm SD \\
\hline Nutrition behaviour & 9 & $22.29 \pm 4.38$ \\
Physical activities & 8 & $15.27 \pm 4.79$ \\
Stress management & 8 & $20.21 \pm 4.03$ \\
Health responsibilities & 9 & $21.23 \pm 4.62$ \\
Spiritual growth & 9 & $24.47 \pm 4.45$ \\
Interpersonal relation & 9 & $23.31 \pm 4.10$ \\
Overall HPLP-II & 52 & $126.45 \pm 16.91$
\end{tabular}


Table 3. Socioeconomic status related to the HPL profile -II's dimensions

\begin{tabular}{|c|c|c|c|c|c|c|c|c|c|c|c|c|c|c|c|}
\hline \multirow{2}{*}{\multicolumn{2}{|c|}{ Variable }} & \multicolumn{2}{|c|}{$\begin{array}{l}\text { Nutrition } \\
\text { Behavior }\end{array}$} & \multicolumn{2}{|c|}{ Physical Activity } & \multicolumn{2}{|c|}{$\begin{array}{l}\text { Stress Man- } \\
\text { agement }\end{array}$} & \multicolumn{2}{|c|}{$\begin{array}{c}\text { Health } \\
\text { Responsi- } \\
\text { bility }\end{array}$} & \multicolumn{2}{|c|}{ Spiritual Growth } & \multicolumn{2}{|c|}{$\begin{array}{l}\text { Interpersonal } \\
\text { Relation }\end{array}$} & \multicolumn{2}{|c|}{ HPLP-II } \\
\hline & & $\begin{array}{c}\text { Mean } \\
\pm \text { SD }\end{array}$ & $\mathbf{P}$ & Mean \pm SD & $\mathbf{P}$ & $\begin{array}{c}\text { Mean } \pm \\
\text { SD }\end{array}$ & $\mathbf{P}$ & $\begin{array}{c}\text { Mean } \\
\pm \text { SD }\end{array}$ & $\mathbf{P}$ & Mean \pm SD & $\mathbf{P}$ & $\begin{array}{c}\text { Mean } \\
\pm \text { SD }\end{array}$ & $\mathbf{P}$ & $\begin{array}{c}\text { Mean } \\
\pm \text { SD }\end{array}$ & $\mathbf{P}$ \\
\hline \multirow{3}{*}{ 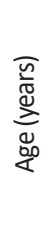 } & $\leq 30$ & $\begin{array}{c}2.43 \pm \\
0.47\end{array}$ & \multirow{3}{*}{$\stackrel{0}{\stackrel{*}{0}}$} & $1.9 \pm 0.59$ & \multirow{3}{*}{$\begin{array}{l}\mathscr{l}_{0} \\
\infty \\
\infty \\
0\end{array}$} & $2.47 \pm 0.45$ & \multirow{3}{*}{$\stackrel{*}{\infty}$} & $\begin{array}{c}2.35 \pm \\
0.51\end{array}$ & \multirow{3}{*}{ 竝 } & $2.68 \pm 0.5$ & \multirow{3}{*}{ 芴 } & $\begin{array}{c}2.58 \pm \\
0.44\end{array}$ & \multirow{3}{*}{ 离 } & $\begin{array}{l}2.41 \pm \\
0.33\end{array}$ & \multirow{3}{*}{ : } \\
\hline & $31-40$ & $\begin{array}{c}2.457 \pm \\
0.51\end{array}$ & & $1.89 \pm 0.6$ & & $2.57 \pm 0.52$ & & $\begin{array}{c}2.36 \pm \\
0.52\end{array}$ & & $2.72 \pm 0.49$ & & $\begin{array}{c}2.55 \pm \\
0.42\end{array}$ & & $\begin{array}{c}2.43 \pm \\
0.34\end{array}$ & \\
\hline & $40<$ & $\begin{array}{c}2.67 \pm \\
0.48\end{array}$ & & $1.87 \pm 0.61$ & & $2.56 \pm 0.53$ & & $\begin{array}{c}2.44 \pm \\
0.53\end{array}$ & & $2.73 \pm 0.51$ & & $\begin{array}{c}2.64 \pm \\
0.52\end{array}$ & & $\begin{array}{c}2.49 \pm \\
0.38\end{array}$ & \\
\hline \multirow{2}{*}{ 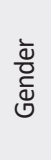 } & Male & $\begin{array}{c}2.41 \pm \\
0.51\end{array}$ & \multirow{2}{*}{$\stackrel{*}{*}{ }^{*}$} & $1.97 \pm 0.61$ & \multirow{2}{*}{$\stackrel{*}{n}$} & $2.51 \pm 0.52$ & \multirow{2}{*}{$\begin{array}{l}\infty \\
\infty \\
0 \\
0 \\
0\end{array}$} & $\begin{array}{c}2.34 \pm \\
0.53\end{array}$ & \multirow{2}{*}{ 음 } & $2.73 \pm 0.51$ & \multirow{2}{*}{ 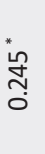 } & $\begin{array}{c}2.6 \pm \\
0.5\end{array}$ & \multirow{2}{*}{ : } & $\begin{array}{c}2.43 \pm \\
0.37\end{array}$ & \multirow{2}{*}{$\begin{array}{l}\text { 0] } \\
\text { on } \\
0\end{array}$} \\
\hline & Female & $\begin{array}{l}2.55 \pm \\
0.45\end{array}$ & & $1.81 \pm 0.57$ & & $2.54 \pm 0.47$ & & $\begin{array}{c}2.42 \pm \\
0.49\end{array}$ & & $2.67 \pm 0.49$ & & $\begin{array}{c}2.57 \pm \\
0.4\end{array}$ & & $\begin{array}{c}2.44 \pm \\
0.32\end{array}$ & \\
\hline \multirow{3}{*}{ 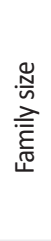 } & $\leq 3$ & $\begin{array}{c}2.39 \pm \\
0.49\end{array}$ & \multirow{3}{*}{ 苑 } & $1.83 \pm 0.56$ & \multirow{3}{*}{ 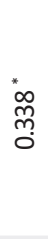 } & $2.54 \pm 0.5$ & \multirow{3}{*}{ 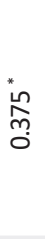 } & $\begin{array}{c}2.37 \pm \\
0.5\end{array}$ & & $2.75 \pm 0.52$ & & $\begin{array}{c}2.59 \pm \\
0.44\end{array}$ & & $\begin{array}{c}2.42 \pm \\
0.34\end{array}$ & \\
\hline & $4-5$ & $\begin{array}{c}2.49 \pm \\
0.48\end{array}$ & & $1.94 \pm 0.6$ & & $2.55 \pm 0.5$ & & $\begin{array}{c}2.38 \pm \\
0.5\end{array}$ & 今. & $2.73 \pm 0.44$ & ్ֶָర & $\begin{array}{l}2.6 \pm \\
0.42\end{array}$ & 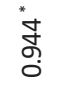 & $\begin{array}{c}2.46 \pm \\
0.32\end{array}$ & $\stackrel{*}{\infty}$ \\
\hline & $6<$ & $\begin{array}{c}2.54 \pm \\
0.51\end{array}$ & & $1.9 \pm 0.62$ & & $2.47 \pm 0.5$ & & $\begin{array}{c}2.36 \pm \\
0.5\end{array}$ & & $2.66 \pm 0.54$ & & $\begin{array}{c}2.58 \pm \\
0.51\end{array}$ & & $\begin{array}{c}2.43 \pm \\
0.38\end{array}$ & \\
\hline$\stackrel{n}{z}$ & Single & $\begin{array}{c}2.46 \pm \\
0.48\end{array}$ & & $1.88 \pm 0.62$ & & $2.46 \pm 0.46$ & & $\begin{array}{c}2.31 \pm \\
0.49\end{array}$ & & $2.62 \pm 0.51$ & & $\begin{array}{c}2.58 \pm \\
0.48\end{array}$ & & $\begin{array}{c}2.39 \pm \\
0.34\end{array}$ & \\
\hline$\frac{\frac{\pi}{n}}{\frac{\pi}{\pi}}$ & Married & $\begin{array}{c}2.47 \pm \\
0.5\end{array}$ & ঙे & $1.9 \pm 0.58$ & ষ্ণ & $2.54 \pm 0.51$ & ָั) & $\begin{array}{c}2.39 \pm \\
0.53\end{array}$ & 芯 & $2.74 \pm 0.49$ & $\begin{array}{l}0 \\
\text { L̊ } \\
0\end{array}$ & $\begin{array}{c}2.59 \pm \\
0.44\end{array}$ & $\underset{\substack{n \\
m \\
o}}{0}$ & $\begin{array}{c}2.45 \pm \\
0.35\end{array}$ & ले \\
\hline$\Sigma$ & $\begin{array}{l}\text { Divorced } \\
\text { /widow }\end{array}$ & $\begin{array}{l}2.67 \pm \\
0.36\end{array}$ & & $1.64 \pm 0.59$ & & $2.46 \pm 0.44$ & & $\begin{array}{l}2.42 \pm \\
0.48\end{array}$ & & $2.63 \pm 0.38$ & & $\begin{array}{c}2.39 \pm \\
0.5\end{array}$ & & $\begin{array}{l}2.4 \pm \\
0.32\end{array}$ & \\
\hline & Doctor & $\begin{array}{l}2.46 \pm \\
0.45\end{array}$ & & $1.86 \pm 0.52$ & & $2.46 \pm 0.57$ & & $\begin{array}{c}2.27 \pm \\
0.6\end{array}$ & & $2.89 \pm 0.68$ & & $\begin{array}{l}2.75 \pm \\
0.65\end{array}$ & & $\begin{array}{c}2.46 \pm \\
0.43\end{array}$ & \\
\hline .̃̃ & Nurse & $\begin{array}{c}2.45 \pm \\
0.49\end{array}$ & in & $1.97 \pm 0.62$ & & $2.55 \pm 0.51$ & $\stackrel{\infty}{*}$ & $\begin{array}{c}2.39 \pm \\
0.48\end{array}$ & 菊 & $2.68 \pm 0.5$ & ${ }_{\infty}^{*}$ & $\begin{array}{c}2.53 \pm \\
0.44\end{array}$ & * & $\begin{array}{l}2.44 \pm \\
0.35\end{array}$ & $\stackrel{*}{*}$ \\
\hline 这 & Lab & $\begin{array}{c}2.46 \pm \\
0.54\end{array}$ & 0 & $1.72 \pm 0.63$ & $\circ$ & $2.46 \pm 0.42$ & 0 & $\begin{array}{c}2.31 \pm \\
0.52\end{array}$ & $0^{\circ}$ & $2.7 \pm 0.44$ & Oㅁ & $\begin{array}{c}2.56 \pm \\
0.45\end{array}$ & ○ं & $\begin{array}{c}2.38 \pm \\
0.32\end{array}$ & 0 \\
\hline & Others & $\begin{array}{c}2.51 \pm \\
0.5\end{array}$ & & $1.88 \pm 0.57$ & & $2.53 \pm 0.5$ & & $\begin{array}{l}2.4 \pm \\
0.53\end{array}$ & & $2.7 \pm 0.46$ & & $\begin{array}{c}2.61 \pm \\
0.42\end{array}$ & & $\begin{array}{c}2.45 \pm \\
0.34\end{array}$ & \\
\hline$\frac{\bar{n}}{\frac{n}{d \pi}}$ & $\leq 5$ & $\begin{array}{c}2.45 \pm \\
0.47\end{array}$ & & $1.93 \pm 0.6$ & & $2.5 \pm 0.44$ & & $\begin{array}{c}2.35 \pm \\
0.49\end{array}$ & & $2.74 \pm 0.49$ & & $\begin{array}{c}2.62 \pm \\
0.44\end{array}$ & & $\begin{array}{c}2.44 \pm \\
0.32\end{array}$ & \\
\hline प्ष & $6-10$ & $\begin{array}{c}2.45 \pm \\
0.54\end{array}$ & 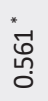 & $1.81 \pm 0.57$ & $\stackrel{*}{\infty}$ & $2.48 \pm 0.55$ & 芦 & $\begin{array}{c}2.31 \pm \\
0.51\end{array}$ & 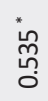 & $2.64 \pm 0.51$ & 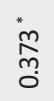 & $\begin{array}{c}2.48 \pm \\
0.46\end{array}$ & $\begin{array}{l}* \\
i_{0}^{*} \\
0 \\
0\end{array}$ & $\begin{array}{l}2.37 \pm \\
0.35\end{array}$ & $\begin{array}{l}\text { : } \\
\stackrel{0}{2} \\
0\end{array}$ \\
\hline $\begin{array}{l}\text { 產 } \\
\text { 음 }\end{array}$ & +11 & $\begin{array}{c}2.51 \pm \\
0.49\end{array}$ & & $1.91 \pm 0.6$ & & $2.55 \pm 0.53$ & & $\begin{array}{c}2.39 \pm \\
0.53\end{array}$ & & $2.72 \pm 0.49$ & & $\begin{array}{c}2.63 \pm \\
0.45\end{array}$ & & $\begin{array}{c}2.46 \pm \\
0.35\end{array}$ & \\
\hline$\overline{\mathrm{d}}$ & Diploma & $\begin{array}{c}2.49 \pm \\
0.5\end{array}$ & & $1.9 \pm 0.62$ & & $2.52 \pm 0.49$ & & $\begin{array}{c}2.36 \pm \\
0.51\end{array}$ & & $2.68 \pm 0.48$ & & $\begin{array}{c}2.57 \pm \\
0.44\end{array}$ & & $\begin{array}{l}2.43 \pm \\
0.35\end{array}$ & \\
\hline . & Bachelor & $\begin{array}{l}2.4 \pm \\
0.48\end{array}$ & 茎 & $1.9 \pm 0.49$ & ᄋ్ & $2.52 \pm 0.48$ & 莳 & $\begin{array}{c}2.37 \pm \\
0.52\end{array}$ & $\begin{array}{l}\stackrel{0}{0} \\
\stackrel{\infty}{0}\end{array}$ & $2.84 \pm 0.55$ & 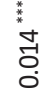 & $\begin{array}{c}2.69 \pm \\
0.52\end{array}$ & $\begin{array}{l}* \\
\% \\
0 \\
0\end{array}$ & $\begin{array}{c}2.46 \pm \\
0.34\end{array}$ & "تี \\
\hline 훔 & $\begin{array}{c}\mathrm{MSc}+ \\
\mathrm{PhD}\end{array}$ & $\begin{array}{c}2.63 \pm \\
0.45\end{array}$ & & $1.86 \pm 0.66$ & & $2.57 \pm 0.68$ & & $\begin{array}{c}2.49 \pm \\
0.68\end{array}$ & & $2.75 \pm 0.64$ & & $\begin{array}{l}2.7 \pm \\
0.57\end{array}$ & & $\begin{array}{c}2.51 \pm \\
0.42\end{array}$ & \\
\hline . 옴 & Satisfied & $\begin{array}{c}2.51 \pm \\
0.55\end{array}$ & & $1.9 \pm 0.61$ & & $2.57 \pm 0.53$ & & $\begin{array}{c}2.35 \pm \\
0.57\end{array}$ & & $2.77 \pm 0.55$ & & $\begin{array}{l}2.6 \pm \\
0.46\end{array}$ & & $\begin{array}{c}2.46 \pm \\
0.38\end{array}$ & \\
\hline 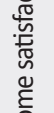 & $\begin{array}{l}\text { Some- } \\
\text { how } \\
\text { satisfied }\end{array}$ & $\begin{array}{c}2.43 \pm \\
0.47\end{array}$ & $\stackrel{\text { m }}{\stackrel{\infty}{0}}$ & $1.91 \pm 0.59$ & ஜூ & $2.48 \pm 0.5$ & ㅇํㅇ & $\begin{array}{c}2.36 \pm \\
0.48\end{array}$ & $\begin{array}{l}* \\
0 \\
0 \\
0 \\
0\end{array}$ & $2.7 \pm 0.44$ & 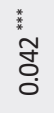 & $\begin{array}{c}2.58 \pm \\
0.45\end{array}$ & 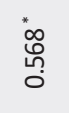 & $\begin{array}{c}2.42 \pm \\
0.34\end{array}$ & 宛 \\
\hline$\underline{\underline{G}}$ & $\begin{array}{l}\text { Notsatis- } \\
\text { fied }\end{array}$ & $\begin{array}{c}2.48 \pm \\
0.43\end{array}$ & & $1.8 \pm 0.59$ & & $2.49 \pm 0.43$ & & $\begin{array}{c}2.41 \pm \\
0.48\end{array}$ & & $2.6 \pm 0.5$ & & $\begin{array}{c}2.54 \pm \\
0.43\end{array}$ & & $\begin{array}{c}2.39 \pm \\
0.31\end{array}$ & \\
\hline
\end{tabular}

* Not Significant; ** Approaching significance; *** Statistically significant 
Hosseini, Ashktorab \& Taghdisi 2013; Mirghafourvand et al. 2014; Panebianco-Warrens, Fletcher \& Kreutz 2014; Chiou, Hsu \& Hung 2016). These results disclosed a statistically significant correlation between age and NB and were compatible with the investigation conducted among Iranian undergraduate students of health (Shariferad et al. 2013).

The present study illustrated that increasing age improved the awareness for NB, HR, SG, and IR with respect to overall HPLP-II. On the contrary, increasing age decreased the physical activities. Thus, the current result was similar to that obtained from previous studies, which postulated that increasing age HPL (Chen, James \& Wang 2007; Huang, Li \& Tang 2010; Mirghafourvand et al. 2014).

This study also presented that females exhibited significantly better behavior in terms of NB, SM, HR, and whole HPLP-II, while the males were better in PA, SG, and IR. These findings were in line with other studies that found sophisticated health-promoting behaviors among females. Two different studies (Panebianco-Warrens, Fletcher \& Kreutz 2014; Kreutz 2008) conducted among music students revealed that female students displayed health-promoting behavior as well as significantly higher score for NB as compared to the male students. In contrast to the current findings, the studies conducted by Alkhawaldeh (2013) and Vançelik et al. (2007) revealed that the average score of NB was higher in males than the females; it was not significant in first one, while it was significant in the second (Vançelik et al. 2007; Alkhawaldeh 2014). Some previous studies did not present significant differences in HPLP-II scores between females and males (Wei et al. 2011; Mirghafourvand et al. 2014; Nacar et al. 2014).

Intriguingly, a sedentary lifestyle is a challenge in the majority of the countries. PA is one of the areas that could be affected by culture, which further limits the of outdoor facilities by females for specific types of PA. Hence, PA had a lower score in females as compared to males; for instance, Iranian women (Baheiraei et al., 2013), academic staff in Turkey (Pirincci et al. 2008), and nursing students in Kuwait (Al-Kandari, Vidal \& Thomas 2008).

Interestingly, only a slight statistically significant relationship was established between the number of family members and NB. A moderate number in the household exhibited high scores for PA, SM, HR, and IR with overall HPLP-II as compared to a small or large family. A small family has high scores for SG, while a crowded family displays high scores for NB. These results were contradic- tory to those from a previous study; overcrowded household was an obstacle to the individual's ability to make healthy choices and practice HPL (Baheiraei et al. 2013).

The K.HcPrs were married, thereby reporting high scores for health-promoting behavior "PA, SM, SG, IR" than single as well as divorced/widowed HcPrs; conversely, divorced/widowed K.HcPs presented high scores for NB and HR. Similar to our findings, AlKandari, Vidal \& Thomas (2008) conducted a study in Kuwaiti nursing students and illustrated that healthy lifestyle behavior of single individuals was lower than that of married individuals (Al-Kandari, Vidal \& Thomas 2008). Furthermore, according to a study by Hosseini et al. (2014), conducted on Iranian nursing students in Tehran, revealed that the mean scores of PA and SM in unmarried students are more than that in married students.

The finding regarding the expertise of HcPrs revealed a marginally significant correlation between occupation and health-promoting behavior. Also, the current study disclosed that doctors had high mean scores for SG and IR than nurses and other specialists, while the nurses had high mean scores in terms of PA and SM. Another study by Vlismas, Stavrinos \& Panagiotakos (2009) revealed that developed occupational status provides access to health-related services and good nutrition (Vlismas, Stavrinos \& Panagiotakos 2009).

Another criterion of HPLP-II was job experience. In this regard, the study showed that the HcPrs with vast experiences exhibited high mean scores for NB, SM, HR, IR, as well as, overall HPLP-II than those with fewer experiences. Thus, work experience improved the health-promoting behaviors. This finding was in agreement with the results of a study conducted among Turkish workers (Beser, Bahar \& Buyakkaya 2007).

This study found a statistically significant association between education levels and SG, as well as, a slight trend towards significance between education levels and IR. Additionally, the post-graduation HcPrs reported high mean scores for NB, SM, HR, SG, and IR as compared to graduation. Conversely, the graduate HcPrs showed high mean scores for PA. Thus, with increasing level of education, the total score of HPL and that of the individual subscales were improved as education is one of the HPL predictors. This result was similar to a study conducted among Turkish female nursing students (Kara 2015). However, according to Cockerham (2007), the education levels did not play critical roles in healthy lifestyle practices. 
Finally, the present study provided valuable information about HPL behaviors among K.HcPrs. Nevertheless, the following limitations should be considered when interpreting the findings. The cross-sectional design of the study precluded the ability to infer causal relationships between the statistically significant correlations. Also, the use of a self-reporting questionnaire for measuring the healthcare providers' HPL was another limitation.

In conclusion, this study assessed the health-promoting behaviors among Iraqi K.HcPrs, which scored the highest mean value in SG and lowest in PA. The low PA might be attributed to the high workload, low leisure times, and lack of enabling environment due to the political instability in Kurdistan. On the other hand, the high score for SG could be as a result of the increase in the level of spirituality and religious beliefs, which is an integral part of the daily life of the Kurdish society. Unhealthy lifestyle is associated with several health consequences that continue to gain ground even among health professionals. Therefore, we recommend interdisciplinary interventions to improve the knowledge and public health awareness regarding unhealthy lifestyle and the associated burden. However, the current findings served as a basis for planning health-promoting interventions in Kurdistan, especially for healthcare providers.

\section{Acknowledgments}

We thank all the participants for taking the time to share a piece of their lives with us. The authors would also like to thank the Directorate of Health/ Garman Administrative for co-operation in this study. We would also like to express our great appreciation for the invaluable assistance of Tara Hassan. The study was supported by a grant from the Tehran University of Medical Sciences “TR.TUMS.REC.1394.1793/January 31 31. $2016 ”$.

\section{Conflict of Interest}

The authors declare no conflicts of interest.

\section{References}

Al Kandari, F., Vidal, V. L. \& Thomas, D., 2008. Health-promoting lifestyle and body mass index among College of Nursing students in Kuwait: A correlational study. Nursing $\mathcal{E}$ Health Sciences, 10(1), pp. 43-50. [DOI:10.1111/j.14422018.2007.00370.x]
Alkhawaldeh, O., 2013. Health promoting lifestyles of Jordanian university students. International Journal of Advanced Nursing Studies, 3(1). [DOI:10.14419/ijans.v3i1.1931]

Baheiraei, A. et al., 2013. Health-promoting behaviors and social support in Iranian women of reproductive age: A sequential explanatory mixed methods study. International Journal of Public Health, 59(3), pp. 465-73. [DOI:10.1007/s00038-013-0513-y]

Beser, A., Bahar, Z. \& Buyakkaya, D., 2007. Health promoting behaviors and factors related to lifestyle among Turkish workers and occupational health nurses' responsibilities in their health promoting activities. Industrial Health, 45(1), pp. 151-9. [DOI:10.2486/indhealth.45.151]

Chen, M. Y., James, K. \& Wang, E. K., 2007. Comparison of health-promoting behavior between Taiwanese and American adolescents: A cross-sectional questionnaire survey. International Journal of Nursing Studies, 44(1), pp. 59-69. [DOI:10.1016/j.ijnurstu.2005.11.015]

Chiou, A. F., Hsu, S. P. \& Hung, H. F., 2016. Predictors of health-promoting behaviors in Taiwanese patients with coronary artery disease. Applied Nursing Research, 30, pp. 1-6. [DOI:10.1016/j.apnr.2015.08.008]

Cockerham, W. C., 2007. Social causes of health and disease. Cambridge: Polity. [PMCID]

Fahimen, S. S., et al., 2007. The review on lifestyle and its relation with the pregnancy outcomes in the pregnant women coming to educational hospitals of Tabriz in 2004. Research Journal of Medical Sciences, 1(2), pp. 91-4.

Ginsborg, J. et al., 2009. Healthy behaviours in music and nonmusic performance students. Health Education, 109(3), pp. 242-58. [DOI:10.1108/09654280910955575]

Hosseini, M. et al., 2014. Health-promoting behaviors and their association with certain demographic characteristics of nursing students of Tehran City in 2013. Global Journal of Health Science, 7(2). [DOI:10.5539/gjhs.v7n2p264]

Hosseini, M., Ashktorab, T., \& Taghdisi, M. H., 2013. Health promotion lifestyle in nursing students: A systematic review. Journal of Health Promotion Management, 2(1), pp. 66-79.

Huang, S. L., Li, R. H. \& Tang, F. C., 2010. Comparing disparities in the health-promoting lifestyles of Taiwanese workers in various occupations. Industrial Health, 48(3), pp. 256-64. [DOI:10.2486/indhealth.48.256]

Kamali, A. S. M. A., et al., 2016. Reliability and validity of Kurdish language version of health promoting lifestyle profile II among Kurdish healthcare providers Kurdish version of HPLP-II. Archives of Iranian Medicine, 19(12): pp. 824-31. [PMID]

Kara, B., 2015. The efficacy of an educational intervention on health behaviors in a sample of Turkish female nursing students: A longitudinal, quasi-experimental study. Nurse Education Today, 35(1), pp. 146-51. [DOI:10.1016/j.nedt.2014.08.015]

Kim, S. H., 2009. Older people's expectations regarding ageing, health-promoting behaviour and health status. Journal of Advanced Nursing, 65(1), pp. 84-91. [DOI:10.1111/j.13652648.2008.04841.x]

Kreutz, G., 2008. Music students' health problems and healthpromoting behaviours. Medical Problems of Performing Artists, 23(1), pp. 3-11. 
Lee, F. H. \& Wang, H. H., 2005. A preliminary study of a healthpromoting lifestyle among Southeast Asian women in Taiwan. The Kaohsiung Journal of Medical Sciences, 21(3), pp. 11420. [DOI:10.1016/S1607-551X(09)70287-6]

Mirghafourvand, M. et al., 2014. A population-based study of health-promoting behaviors and their predictors in Iranian women of reproductive age. Health Promotion International, 30(3), pp. 586-94. [DOI:10.1093/heapro/dat086]

Mirghafourvand, M., Sehhati, F., \& Rahimi, M., 2014. Healthpromoting lifestyle and its demographic predictors in infertile couples referred to infertility clinic of tabriz al-zahra hospital, 2013. Journal of caring sciences, 3(3), pp. 175-84. doi: 10.5681/ jcs.2014.019

Nacar, M. et al., 2014. Health promoting lifestyle behaviour in medical students: A multicentre study from Turkey. Asian Pacific Journal of Cancer Prevention, 15(20), pp. 8969-74. [DOI:10.7314/APJCP.2014.15.20.8969]

Panebianco Warrens, C. R., Fletcher, L. \& Kreutz, G., 2014 Health-promoting behaviors in South African music students: A replication study. Psychology of Music, 43(6), pp. 779-92. [DOI:10.1177/0305735614535829]

Peker, K., \& Bermek, G., 2011. Predictors of health-promoting behaviors among freshman dental students at Istanbul University. Journal of dental education, 75(3), pp. 413-20. [PMID]

Pender, N., Murdaugh, C. \& Parsons, M., 2002. The health promotion model. Health Promotion in Nursing Practice (pp. 59-79). New York: Appleton \& Lange.

Pirincci, E. et al., 2008. Factors affecting health-promoting behaviours in academic staff. Public Health, 122(11), pp. 1261-3. [DOI:10.1016/j.puhe.2008.04.006]

Schopp, L. H. et al., 2015. Act healthy: Promoting health behaviors and self-efficacy in the workplace. Health Education Research, 30(4), pp. 542-53. [DOI:10.1093/her/cyv024]

Scott, D. \& Happell, B., 2011. The high prevalence of poor physical health and unhealthy lifestyle behaviours in individuals with severe mental illness. Issues in Mental Health Nursing, 32(9), pp. 589-97. [DOI:10.3109/01612840.2011.569846]

Shariferad, G. et al., 2013. Health-promoting lifestyle and quality of life among undergraduate students at school of health, Isfahan university of medical sciences. Journal of Education and Health Promotion, 2(1), p. 11. [DOI:10.4103/2277-9531.108006]

Sousa, P. et al., 2014. Measuring health-promoting behaviors: Cross-cultural validation of the health-promoting lifestyle profile-II. International Journal of Nursing Knowledge, 26(2), pp. 54-61. [DOI:10.1111/2047-3095.12065]

Steyn, K., \& Damasceno, A., 2006. Lifestyle and related risk factors for chronic diseases. Disease and Mortality in Sub-Saharan Africa, 2, pp. 247-65.

Vançelik, S., et al., 2007. Nutrition knowledge and habits and associated factors in University Students. TSK Preventive Medicine Bulletin, 6, pp. 242-8.

Vlismas, K., Stavrinos, V., \& Panagiotakos, D. B., 2009. Socioeconomic status, dietary habits and health-related outcomes in various parts of the world: A review. Central European Journal of Public Health, 17(2), p. 55-63. [PMID]
Wallace, K., 2000. Determinants of health promoting behaviors in older adults [MSc. thesis]. Allendale Charter Township, Michigan: Grand Valley State University.

Wei, C. N. et al., 2011. Assessment of health-promoting lifestyle profile in Japanese university students. Environmental Health and Preventive Medicine, 17(3), pp. 222-7. [DOI:10.1007/s12199011-0244-8]

World Health Organization, 2005. The Bangkok charter for health promotion in a globalized world. Geneva: World Health Organization.

World Health Organization, 2015a. Fact sheets: Non-communicable diseases. Geneva: World Health Organization.

World Health Organization, 2015b. Health topics: Physical activity. Geneva: World Health Organization. 